\title{
LE AGENDE GLOBALI DI SVILUPPO DELLE NAZIONI UNITE
}

\author{
ENRICA CHIAPPERO (*) \\ Nota presentata dal m.e. Silvio Beretta
}

(Adunanza del 9 giugno 2016)

SunTO. - Negli ultimi due decenni è cresciuta la consapevolezza da parte della comunità internazionale, o quanto meno di una larga parte di essa, di affrontare con urgenza quelle sfide globali che, tanto i paesi industrializzati quanto quelli in via di sviluppo, si trovano oggi ad affrontare. Questioni quali i cambiamenti climatici, i movimenti migratori, le diseguaglianze crescenti e le povertà persistenti pongono, nei fatti, seri dubbi sulla sostenibilità sociale, economica ed ambientale dell'attuale modello di sviluppo e richiedono un impegno serio e, per quanto possibile, comune. E' a partire anche da queste premesse, che le Nazioni Unite hanno promosso due agende globali di sviluppo, rispettivamente gli Obiettivi del Millennio nel 2000 (MDGs) e gli Obiettivi di Sviluppo Sostenibile nel 2015 (SDGs), fissando obiettivi e traguardi specifici da raggiungere. E' ragionevole chiedersi se può essere questa la modalità ottimale o adeguata ad affrontare queste sfide e se, o in che misura, quanto si è appreso dall'esperienza della prima agenda sia stato di aiuto nella preparazione della seconda. Questa breve nota toccherà queste questioni ponendo in confronto le due agende.

$$
* * *
$$

ABSTRACT. - In the last two decades the international community (or at least part of it) has shown an increasing awareness regarding the global challenges that either developed and developing countries are facing with: these challenges, which surely include climate changes and international migrations, growing inequalities and persisting poverty, are raising serious concerns regarding the environmental, social and economic sustainability of the current model of development. On the basis of these arguments the

(") Dipartimento di Scienze Politiche e Sociali, Università di Pavia, Italy.

E-mail: enrica.chiappero@unipv.it 
UN system has promoted two main Agendas for development, in 2000 and 2015, setting goals and targets to be achieved. But do we really need an Agenda for development? What have we learnt from the MDGs and to what extent criticisms and weaknesses in the Agenda 2015 have been taken into account in designing the Agenda 2030? This short note is aimed to briefly touch some of these issues by comparing the two agendas.

La questione su cui vorrei richiamare brevemente l'attenzione è se sia realizzabile un modello di sviluppo globalmente sostenibile sul piano ambientale, oltre che sul fronte sociale ed economico e se, nell'intento di perseguire tale scopo, possa essere di aiuto disporre di una agenda globale di sviluppo condivisa dalla comunità internazionale. Posta in un'altra prospettiva, possiamo chiederci se e in che misura sia possibile passare dall'adesione generale a una visione di intenti e di buone intenzioni, ampiamente e ragionevolmente condivisa, ad un insieme di decisioni e di azioni coordinate e concrete che mirino all'effettiva realizzazione di tale risultato.

La prima domanda su cui riflettere è perché mai dovremmo avere bisogno di una agenda comune. In fondo, possiamo immaginare che ogni paese, ogni comunità aspiri naturalmente allo sviluppo e al progresso, che ogni azione umana abbia come finalità principale il miglioramento della qualità della vita e del benessere individuale e che, tendenzialmente, nessuno si auguri un arretramento delle condizioni di vita proprie o altrui, o del benessere della comunità cui appartiene. È dai tempi di Adam Smith che siamo abituati a pensare che, grazie alla somma di azioni che mirano al perseguimento del proprio interesse personale, si ottiene il massimo sviluppo e benessere per la società nel suo complesso, fine ultimo verso cui le nazioni dovrebbero tendere.

Oggi potremmo dire, però, che una delle ragioni principali per cui la somma di azioni individuali non è più sufficiente, ed è necessaria, piuttosto, l'adesione a un progetto comune, è che la gran parte delle sfide che ci troviamo oggi ad affrontare sono sfide globali che difficilmente potranno trovare una qualche forma di soluzione se non verranno ricomprese in una prospettiva più ampia, che vada al di là del singolo attore, della singola comunità, del singolo paese, delle singole generazioni. Le questioni sul tavolo sono ben note: comprendono il tema dei cambiamenti climatici e, più in generale, la tutela dell'ambiente e la sostenibilità dei nostri modelli di sviluppo. Riguardano le forme estreme, ancora assai diffuse di 
povertà, le crescenti disuguaglianze che esistono all'interno delle comunità e i perduranti divari tra nord e sud del mondo, gli effetti che questi divari producono sul fronte dell'instabilità politica e sociale, delle migrazioni, delle prospettive di pace. Includono la salute globale e la persisten$\mathrm{za}$, in alcune regioni, di malattie in larga misura debellate in altre parti del mondo, i rischi di epidemie e la loro rapida diffusione in una comunità umana globalizzata. Sono legati all'innovazione tecnologica e allo sviluppo digitale, alle crescenti opportunità che questa rivoluzione digitale comporta, ma anche all'imprevedibilità dei rischi e all'uso di queste tecniche, con la necessaria difesa dei dati personali e la regolamentazione nell'uso di tali dati su scala ampia. Opportunità, sfide, rischi di questa natura richiedono una visione globale e complessa e dunque legittimano il tentativo di disporre di un'agenda di lavoro che indirizzi le azioni della comunità internazionale.

In effetti, tentativi di questo genere sono stati compiuti in anni relativamente recenti. Tra questi, sicuramente uno dei più importanti è la dichiarazione di Rio de Janeiro su ambiente e sviluppo, in occasione del summit delle Nazioni Unite del 1992: su questa base venne definita l'Agenda 21, un'agenda globale per il ventunesimo secolo, a cui hanno fatto seguito analoghi documenti predisposti a livello nazionale. Nell'alveo di queste esperienze si inserisce nel 2000 la UN Millennium Declaration, sulla base della quale le Nazioni Unite hanno fissato l'Agenda 2015 e i corrispondenti Millennium Development Goal, su cui torneremo a parlare tra poco. Ancor più di recente formulazione, è l'Agenda 2030 in vigore dal $1^{\circ}$ gennaio 2016. Questa fase di passaggio tra le due agende globali è di particolare interesse ai nostri fini perché ci consente di tracciare un primo bilancio nei confronti degli obiettivi, raggiunti o falliti, compresi nell'esperienza appena conclusa, ma anche di guardare alle sfide e alle priorità che la comunità internazionale si è posta per i prossimi 15 anni. È, soprattutto, un'occasione interessante di riflessione su ciò che ha funzionato e ciò che ha fallito nell'Agenda del nuovo millennio, sugli errori commessi e su quelli corretti, sulla capacità di affrontare i problemi mai risolti - come l'eliminazione della povertà o della fame - e le nuove sfide poste alle nostre società dalla rivoluzione digitale ai cambiamenti climatici.

In cosa consistono queste Agende? Si tratta, nello specifico, di documenti che non si limitano a delineare scenari, esprimere auspici o suggerire possibili linee di azione, come è tipico delle dichiarazioni universali. Molto più pragmaticamente, definiscono specifici obiettivi di 
sviluppo in ambiti di interesse prioritario per la comunità internazionale (come povertà, fame, salute o istruzione), fissano specifici traguardi da realizzare (come dimezzare la povertà o eliminare la fame) e individuano un insieme di indicatori che permettano di misurarne la consistenza e monitorarne i progressi (come la quota di poveri sulla popolazione totale o la percentuale di persone malnutrite o sottonutrite). La prima Agenda, appena portata a compimento, poneva otto obiettivi di sviluppo, articolati in 21 target da realizzare entro il 2015, misurati attraverso 60 indicatori specifici. La nuova Agenda segue un'impostazione simile, ma si presenta più ampia ed ambiziosa in termini di risultati da realizzare entro il 2030. Gli obiettivi, infatti, sono saliti a 17, i target a 169 e circa 240 sono gli indicatori previsti.

La prima Agenda, decisamente più essenziale ma non per questo di più facile realizzazione, richiamava l'attenzione su questioni sociali urgenti come: sradicare la povertà estrema e la fame, primo obiettivo su cui maggiormente si è concentrata l'attenzione; rendere universale l'istruzione primaria, garantendo in egual misura a bambini e bambine l'accesso alla scuola; promuovere l'uguaglianza di genere rafforzando la posizione e la capacità di azione delle donne; ridurre la mortalità materna e tutelare la salute materna; combattere malattie di carattere endemico, in particolare l'AIDS, ma anche la malaria e altre malattie curabili che colpiscono ancora oggi molti paesi in via di sviluppo, con serie conseguenze dal punto di vista della mortalità, soprattutto infantile; preservare l'ambiente e rendere sostenibili i processi di sviluppo; definire strategie e partnership globali per favorire lo sviluppo.

Gli obiettivi e target specifici definiti nell'Agenda per il nuovo millennio hanno rappresentato un punto di riferimento importante per la cooperazione internazionale, un richiamo alla responsabilità collettiva per affrontare e cercare di debellare problemi mai risolti, come la povertà estrema e la fame, che non possono più trovare giustificazione plausibile nel XXI secolo. Hanno affermato la necessità di intraprendere uno sforzo comune in questa direzione da parte dei governi, delle agenzie di sviluppo, delle organizzazioni non governative. Sono serviti da guida per i paesi in via di sviluppo e per i donatori internazionali per individuare politiche e azioni finalizzate e coerenti alla realizzazione di questi traguardi. Ma hanno anche posto in luce l'importanza di sensibilizzare la società civile, le scuole, i media, le generazioni più giovani nei rispetti di queste sfide globali. L'evento dell'EXPO e la Carta di Milano sono un esempio significativo in tal senso. 
Giunti a conclusione di questo percorso, è oggi tempo di bilanci, per quanto sia difficile e complesso misurare se, quali e quanti target siano stati - intenzionalmente o involontariamente - realizzati, tanto più in circostanze in cui intervengono fattori non previsti, sul piano economico e politico, che influenzano o modificano le traiettorie in direzione positiva o negativa. È opinione generale che l'Agenda per il nuovo millennio sia stata una storia di relativo successo: alcuni obiettivi importanti sono stati effettivamente raggiunti: per esempio quello di dimezzare la povertà estrema e di garantire eguaglianza di genere alla scuola primaria: di fatto, oltre il 90\% dei bambini e delle bambine oggi al mondo ha accesso almeno alla scuola elementare. Buoni progressi sono stati anche realizzati in altri ambiti, in particolare nel campo della mortalità infantile, nella prevenzione dell'HIV o della tubercolosi e della malaria. Altri traguardi sono però ancora lontani: è il caso della mortalità materna, dell'accesso all'acqua e a servizi igienici adeguati, dell'eguaglianza tra uomini e donne.

Un ulteriore elemento di preoccupazione è legato al fatto che il risultato globale nasconde situazioni assai differenziate al proprio interno. Ad esempio, la realizzazione del primo goal (povertà e fame) su scala mondiale è in larga parte dovuto all'eccezionale performance di paesi come Cina e, in parte, India, la cui dimensione in termini di popolazione è tale da generare immediate ripercussioni (in questo caso positive) sul dato globale. In realtà, nessuno degli otto obiettivi del millennio è stato realizzato in quelli che vengono definiti dalla Banca Mondiale come "paesi fragili", cioè paesi a basso reddito, talvolta in conflitto, a cui si associa una fragilità sostanziale dal punto di vista istituzionale e di stabilità politica. In alcuni paesi, in particolare nell'Africa Sub-Sahariana, molti di questi indicatori sono addirittura peggiorati. Inoltre, quello che si osserva è che la crescita economica, misurata attraverso il tasso di crescita del Prodotto Interno Lordo, non è di per sé sufficiente a garantire una crescita degli indicatori di tipo sociale qui considerati e che non necessariamente esiste una correlazione positiva neppure tra obiettivi. Tra i molti esempi possibili possiamo citare il caso dell'India, la cui crescita economica è stata decisamente positiva nel lasso temporale di riferimento; anche dal punto di vista della lotta alla povertà ha ottenuto risultati molto buoni, senza riuscire tuttavia a risolvere uno dei problemi che storicamente la assilla, come la malnutrizione infantile (quasi il 36\% dei bambini indiani soffre ancora la fame ed è sottopeso). Analogamente, la crescita economica registrata in molti 
paesi emergenti o nei cosiddetti oil countries (paesi produttori ed esportatori di petrolio) non ha necessariamente portato con sé risultati positivi sul fronte, ad esempio, dell'uguaglianza di genere.

Infine, sullo sfondo, resta il problema del costo della realizzazione di questi obiettivi e dunque della sostenibilità finanziaria. La domanda interessante da porre, per quanto di difficile risposta, dovrebbe essere se la disponibilità, per la prima volta, di una Agenda di sviluppo così specifica e definita sia stata in grado di attivare energie, risorse economiche e di attenzione adeguate, o quanto meno sufficienti, a realizzare tali traguardi. Alcune analisi condotte da ricercatori della Banca Mondiale nel 2002 stimavano in circa 50 miliardi di dollari l'anno il fabbisogno aggiuntivo di risorse, sotto forma di aiuti ufficiali allo sviluppo, necessario per la realizzazione di questi obiettivi. All'incirca il doppio rispetto a quanto investito nel 2000 su questo fronte. È opportuno ricordare che i paesi occidentali, con la dichiarazione del millennio, si erano impegnati a destinare lo $0,7 \%$ del loro PIL per gli aiuti allo sviluppo ma, nei fatti, nessun paese o quasi ha onorato questo impegno. In media, i Paesi OCSE hanno destinato a questa finalità lo $0,31 \%$ del PIL, dunque meno della metà, con il nostro paese che si colloca al di sotto della media con un modesto 0,23 per cento del reddito nazionale. L'insufficienza delle risorse messe in gioco potrebbe essere avanzata come una delle cause che può spiegare la performance, non particolarmente esaltante, di quest'Agenda. In realtà, le ragioni del parziale successo o insuccesso non possono essere ascritte solo all'insufficienza di risorse. I paesi che hanno realizzato maggiori progressi non sono necessariamente quelli che hanno ricevuto più aiuti: accanto alle risorse, pur sempre essenziali, occorrono capacità di programmazione e buona gestione amministrativa, istituzioni adeguate che sappiano impiegare al meglio queste risorse concentrandole nella direzione giusta, condizioni queste difficilmente presenti nei paesi in via di sviluppo e, in particolare, nei contesti fragili sopra menzionati.

Al di là della soddisfazione, parziale o meno, rispetto agli esiti raggiunti, sono stati rimarcati altri elementi di debolezza nei confronti di questa prima agenda di sviluppo su scala globale, in particolare in merito alle modalità attraverso cui si è giunti alla sua definizione. È stato, ad esempio, rimarcato il gap esistente tra il contenuto della Dichiarazione del Millennio, con i suoi richiami a princìpi cardine, come i princìpi di libertà, di universalità, di equità, di giustizia, di tolleranza, di solidarietà, e il fatto che questi hanno trovato solo parziale riscontro nell'i- 
dentificazione degli obiettivi. Una seconda critica, evidente, è la scarsa attenzione riservata alle tematiche ambientali ma anche ad alcune sfide importanti che i paesi si trovano oggi ad affrontare, ad esempio, sul mercato del lavoro e sulle relative trasformazioni. Allo stesso modo, si sottolinea che non si è prestata attenzione sufficiente agli aspetti di governance delle istituzioni, al tema della pace e sicurezza, tutti elementi strettamente collegati con le tematiche di sviluppo. Nessuna particolare considerazione è stata riservata neppure al tema delle disuguaglianze: di reddito e di ricchezza, ma anche di risultati e di opportunità, all'interno e tra paesi, e all'interno e tra generazioni. Inoltre, gli MDGs sono stati definiti in termini di target $\mathrm{e}$ indicatori nazionali: si è guardato così ai valori medi riferiti a singoli paesi senza chiedersi in che misura questi esiti fossero rappresentativi delle condizioni effettive di vita della popolazione in quegli stessi paesi. Infine, si è badato soprattutto alla consistenza numerica di questi obiettivi di sviluppo, guardando a quella che era la condizione di partenza e a quello che avrebbe dovuto essere il target di arrivo, ma non vi è stata una sufficiente riflessione sulle cause alla base della prima e sui possibili ostacoli alla loro realizzazione, o sui mezzi e sugli strumenti necessari per superare tali ostacoli.

$\grave{E}$ allora ragionevole chiedersi in che misura questi rilievi critici siano stati di aiuto o presi in considerazione in fase di definizione della seconda Agenda di sviluppo, quella che accompagnerà il percorso di sviluppo fino al 2030. Come si è rimarcato, una delle prime critiche avanzate riguarda la scarsa condivisione dell'Agenda precedente, esito più di una decisione presa a tavolino tra tecnici delle Nazioni Unite, seppur con esperienza e ragionevolezza, che di una discussione aperta e ponderata sulle priorità o sulle aspirazioni dei singoli paesi e delle loro comunità.

Senza dubbio, la procedura adottata per l'identificazione degli obiettivi di sviluppo da includere nella nuova Agenda, con i relativi target, è stata assai diversa dalla precedente. È stato l'esito finale di un lungo percorso avviato nel 2012, con l'istituzione di un gruppo di lavoro aperto, costituito da una rappresentanza di settanta paesi, che ha portato alla redazione di un documento preliminare con l'identificazione dei possibili goal. A questo ha fatto seguito un rapporto del Segretario delle Nazioni Unite all'Assemblea dal titolo The Road to Dignity by 2030: Ending Poverty, Transforming All Lives and Protecting the Planet. Per questa via, e attraverso una serie di ulteriori consultazioni, si è giunti, infine, alla Risoluzione delle Nazioni Unite n. 70/1 Transforming our World: the 2030 Agenda for Sustainable Development 
adottata da 193 paesi ed entrata in vigore dal $1^{\circ}$ gennaio 2016. Oltre a questo percorso istitutivo dell'Agenda, ve ne è un altro, parallelo e ancora in corso, che riguarda l'identificazione del set di indicatori necessari a monitorare l'andamento nel tempo degli obiettivi, indicatori che dovranno soddisfare criteri di comparabilità, affidabilità ed efficienza. È stato inoltre istituito un apposito panel di esperti, denominato High Level Political Forum, che seguirà le fasi di implementazione dell'Agenda, ne osserverà i progressi, suggerirà strategie di azione coerenti a raggiungere il risultato e affronterà eventuali questioni emergenti che potranno di volta in volta presentarsi lungo il percorso.

Con una certa capacità di retorica, pur sempre utile quando si tratta di coalizzare il consenso e catturare l'attenzione dei media e dell'opinione pubblica, le Nazioni Unite identificano in "cinque P" (people, planet, prosperity, peace and partnerships) i princìpi di fondo che hanno ispirato l'Agenda 2030. Questi princìpi sottolineano la necessità di concentrarsi sulle persone (attori e beneficiari di ogni processo di sviluppo), di favorire processi di crescita economica che siano inclusivi e che vadano soprattutto a vantaggio delle fasce più marginali della popolazione, di tutelare e proteggere gli ecosistemi del nostro pianeta, di garantire società sicure, ponendole al riparo da guerre e tensioni sociali e politiche, di favorire una partnership e una solidarietà a livello globale per la realizzazione di questi fini.

Il più ampio spettro di goal e di target, e conseguentemente di indicatori, a cui si è giunti con la nuova Agenda, risponde sicuramente alle critiche avanzate sull'omissione di dimensioni importanti per lo sviluppo: in primo luogo, sicuramente, l'ambiente nei suoi molti aspetti rilevanti, ma anche le disuguaglianze, le istituzioni, la pace. Senza entrare troppo nel dettaglio dei singoli aspetti, è sufficiente porre a confronto le due agende (Fig. 1) per osservare che alcuni obiettivi sono rimasti, seppur ripensati e diversamente articolati, mentre altri del tutto nuovi si sono aggiunti. Resta, come primo obiettivo, l'eliminazione della povertà, intesa questa volta non solo come carenza di reddito ma nelle sue molteplici forme; permane l'impegno a sconfiggere la fame, ma ora considerato come obiettivo a sé stante; ritroviamo l'istruzione e la salute, considerati però ora anche in termini di qualità dei servizi educativi e sanitari forniti; persiste l'intenzione di ridurre i divari di genere, mai risolti nonostante $\mathrm{i}$ buoni progressi raggiunti a livello globale. Sono inclusi, per la prima volta, aspetti importanti trascurati nella precedente Agenda: per esempio l'accesso al mercato del lavoro, non soltanto come opportunità occupa- 
zionale sui generis ma anche come posti di lavoro decenti, sicuri e adeguatamente remunerati. Si rimarca l'importanza di dotare i paesi di infrastrutture di base essenziali per il loro sviluppo, di progettare città e comunità sostenibili e sicure. Ma l'elemento certamente distintivo di questa agenda rispetto alla precedente è l'attenzione nei confronti della questione ambientale. Benché questo tema sia trasversale e presente all'interno di molti dei goal dell'Agenda 2030, ad esempio quando si fa riferimento a modelli di produzione e consumo sostenibili o alla possibilità di accesso a fonti di energia pulita e a basso costo per gli usi domestici quotidiani o, ancora, ad acqua pulita e a servizi igienico-sanitari adeguati, vi sono obiettivi che guardano specificatamente all'ambiente: il riferimento è in particolare ai goal 13-15 dedicati rispettivamente al clima e alla vita, nei mari e sulla terra. Infine, il riconoscimento che non si possono realizzare piani di sviluppo se non è garantita la stabilità politica, se non vi sono istituzioni solide ed efficienti, se non si creano condizioni di pace e di sicurezza nazionale. L'ultimo goal, si richiama alla necessità di pervenire ad una partnership globale per la realizzazione di un'agenda così ambiziosa e anche, come vedremo tra poco, così onerosa.

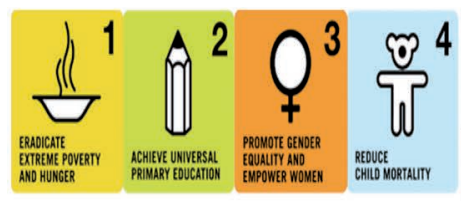

Millennium Development Goals

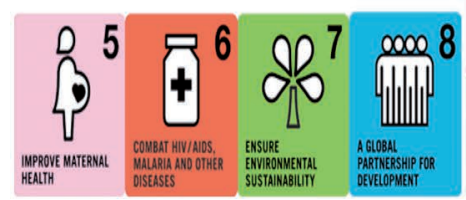

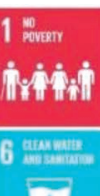
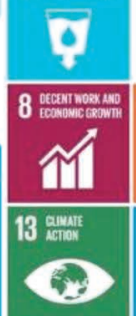
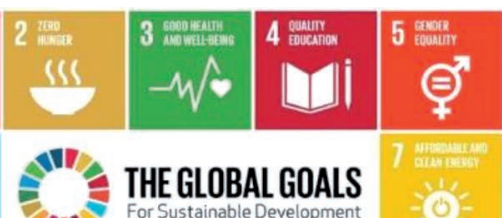

THE GLOBAL GOALS

For Sustainable Development
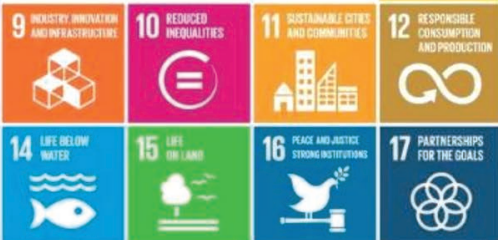

Fig. 1

Vi sono, dunque, importanti similitudini tra le due agende, e certamente la più recente deve molto all'esperienza maturata nel quindicennio precedente, ma altrettante significative differenze. La Tab. 1 qui sotto riportata si limita a mettere in luce solo alcune di queste differenze.

L'Agenda 2030 si presenta come un progetto certamente di ampio 
respiro, con una maggiore articolazione al suo interno, che parla al mondo intero e non solo al sud del mondo, che è stata in qualche misura definita e condivisa a livello globale. $\grave{E}$ anche estremamente complessa e sfidante, e qui si indirizzano, in effetti, le principali critiche già ampiamente avanzate nei suoi confronti. La prima, abbastanza facile e ovvia, è che 17 goal e 169 target sono decisamente troppi e troppo ambiziosi. Questo rischia di rendere assai difficile, se non del tutto improbabile, la realizzazione anche solo di una parte di questi obiettivi, vanificando l'impegno dei paesi e della comunità internazionale. Rischia di spingere i paesi, soprattutto quelli più poveri, a privilegiare scelte e priorità nei confronti di quegli obiettivi più facili da raggiungere, ma probabilmente anche relativamente meno impellenti. Rischia di non saper orientare davvero, come dovrebbe, i piani di sviluppo dei singoli paesi ex ante, quanto piuttosto di giustificarli, in qualche modo, ex post. Qualunque sia la traiettoria di sviluppo di un paese, per quanto modesta, possiamo sempre attenderci che alcuni di questi target saranno realizzati o avvicinati (seppur non sempre con intenzione) e molti altri saranno mancati (non potrebbe che essere così, data la loro numerosità): ma, allora, quale può essere l'utilità di disporre di un'Agenda globale di sviluppo? Infine, rischia di essere "non sostenibile" se la si guarda dal punto di vista della quantità di risorse finanziarie necessarie a realizzarla.

Tab. 1.

\begin{tabular}{|c|c|c|}
\hline Aspetti & MDG & SDG \\
\hline Numero di goal, target, indicatori & $8 / 18 / 48$ & $17 / 169 / 240$ \\
\hline$\underline{\text { Dimensioni considerate }}$ & Sociale & Economica, sociale, ambientale \\
\hline Paesi a cui si rivolge & $\begin{array}{l}\text { Solo paesi in via } \\
\text { di sviluppo }\end{array}$ & Tutti i paesi \\
\hline Procedura & “Top down” & $\begin{array}{l}\text { Più inclusiva (anche se non } \\
\text { "bottom up") }\end{array}$ \\
\hline Standard di riferimento & Medie nazionali & $\begin{array}{l}\text { Oltre la media nazionale } \\
\text { (attenzione alle diseguaglianze) }\end{array}$ \\
\hline Dati statistici & $\begin{array}{l}\text { Statistiche UN } \\
\text { già disponibili }\end{array}$ & $\begin{array}{l}\text { Statistiche UN già disponibili } \\
+ \text { Agenzia ad boc }\end{array}$ \\
\hline Fame e povertà & Unico goal & Goal separati \\
\hline$\underline{\text { Educazione }}$ & "Quantità" & Attenzione alla "qualità" \\
\hline Fonti di finanziamento & $\begin{array}{l}\text { Principalmente } \\
\text { aiuti allo sviluppo }\end{array}$ & $\begin{array}{l}\text { Spettro più ampio di risorse } \\
\text { (incluse risorse private) }\end{array}$ \\
\hline
\end{tabular}


Abbiamo già sottolineato, in precedenza, che le risorse destinate dai paesi ricchi come aiuto pubblico allo sviluppo sono limitate e ben al di sotto degli impegni presi. Possiamo attenderci che un maggior numero di obiettivi da realizzare possa, in qualche modo, attivare maggiori risorse da mettere in gioco? Abbastanza improbabile, vista anche la storia recente. Inoltre, a differenza della precedente, questa Agenda è rivolta a tutti i paesi, anche a quelli industrializzati che dovranno compiere anch'essi sforzi significativi per realizzare, almeno in parte, questi goal, in contesti politici spesso poco lungimiranti. $\mathrm{Ma}$ quante risorse sarebbero necessarie, in via ipotetica, per realizzare l'Agenda 2030? Le Nazioni Unite hanno promosso un summit ad Addis Abeba nel 2015 interamente dedicato alle modalità di finanziamento degli SDGs sottolineando il fatto che gli aiuti pubblici allo sviluppo non possono certo essere sufficienti e che sarà essenziale attivare risorse sia pubbliche sia private a tale scopo, con particolare coinvolgimento delle imprese. Non si sono spinti a fare una stima del loro costo effettivo, stime peraltro sempre assai difficili da fare. Vi è però chi ha cercato di quantificare, seppure in maniera molto approssimativa, l'ammontare di risorse da attivare e queste ammonterebbero, all'incirca, al 4\% del PIL mondiale o al 15\% del risparmio globale. Una quantità enorme di risorse difficilmente destinabile a questo scopo.

Dobbiamo concludere che lo sforzo compiuto dalla comunità internazionale di dotarsi di una propria agenda di sviluppo sia uno sforzo inutile? Certamente no: vi sono buone argomentazioni a sostegno di un progetto di questo tipo. Il primo è che molti dei problemi qui menzionati, in particolare quelli di natura ambientale, sono per loro natura di ordine globale e non potranno mai trovare soluzione da parte di un singolo paese e neppure di un singolo continente. Il secondo è che si è riconosciuta l'importanza di guardare al problema dello sviluppo in prospettiva multidimensionale e complessa, nella consapevolezza che la crescita economica non può essere la chiave di volta del problema dello sviluppo su scala globale e che, al contrario, un'eccessiva spinta nei confronti della crescita economica può generare problemi di insostenibilità sul piano ambientale e su quello sociale. In altre parole, è necessario cambiare orizzonte di riferimento, guardando al medio-lungo periodo, e cambiare prospettiva, considerando accanto allo sviluppo economico anche quello umano. Indubbiamente, se questa è la visione che si accoglie, si corre inevita- 
bilmente il rischio di risultare eccessivamente ambiziosi e di trasformare un'agenda di lavoro in nulla più che un'affascinante utopia. $\mathrm{Ma}$, come ci viene spesso ricordato, se l'utopia, come l'orizzonte è per sua stessa natura irraggiungibile, rappresenta pur sempre uno stimolo per continuare a camminare. 